\title{
A Print-and-scan Resilient Digital Watermark for Card Authentication
}

\author{
Anthony T. S. Ho and Feng Shu \\ School of Electrical and Electronic Engineering \\ Nanyang Technological University \\ Nanyang Avenue, Singapore 639798 \\ E-mail: etsho@ntu.edu.sg
}

\begin{abstract}
In this paper, a secure card authentication system based on digital watermarking is presented. A sequence of identification characters, e.g. owner's ID number is inserted into the photograph, thus a linkage between the photograph and its owner can be established. The print-and-scan process is successfully overcome by our transform coefficient polarity based watermarking algorithm. The retrieval algorithm is capable of verifying whether the inspected card is authorized. We apply our algorithm for $\mathbf{1 0 5}$ photographs and the success rate achieved is better than $96 \%$.
\end{abstract}

\section{INTRODUCTION}

In recent years, the rapid development of digital watermarking has led to a significant number of watermarking algorithms have been proposed in the literature. Apart from copyright protection, which has attracted much research attention, the other application in digital watermarking which has also received a considerable interest is image authentication.

In a watermarking authentication system, a sequence of identification or a visual pattern, that is, the watermark, is imperceptibly embedded into the host media. The watermarked media data generally goes through some distortion channel. The recovered watermark from inspected data is used for authentication in different ways, such as localizing the distortions $([1],[2])$, determining the type of attacks performed [3] or verifying the genuineness of the data.

In this paper, we devise a watermarking system to authenticate cards with the owner's photograph. These cards include student cards, identity cards, passports and driving licenses. Nowadays, with some sophisticated editing tools (for example, Photoshop), and printing and scanning facilities, it is extremely easy to modify or replace the original photograph, and to make fake security documents or cards [6]. It is reported that twothirds of passport frauds are due to photograph substitution [7].

To prevent such photograph substitution frauds, in the proposed watermarking system, we directly establish a linkage between the owner's photo and his/her personal information by imperceptibly superimposing the personal identification information, considered as the watermark, into the original photograph. The main challenge in the proposed system is to overcome the print-and-scan (PS) process, which is regarded as a combination of different attacks [8].
The host data in our watermarking system are face photographs. The size of the standard photos on the security cards, such as student cards, are around $1 \times 1$ inch (we use $180 \times 130$ pixels photos), which is much smaller than the host images used in most image watermarking algorithms proposed in the literature. Face photos are typically very smooth, which are much easier to introduce degradation during watermark embedding. Furthermore, a multi-bit watermark is hidden into the smooth face photos of small size, while the photo integrity must be maintained at the same time.

Our paper is organized as as follows. In section II, we introduce the framework of our proposed card authentication system, the print-and-scan process and the Hadamard transform-based watermarking embedding and retrieval algorithms. Experimental results are presented in section III, and conclusions in section IV.

\section{OUR PROPOSED CARD AUTHENTICATION SYSTEM}

\section{A. Framework of card authentication system}

In this section, the framework of our proposed authentication system is introduced. The watermark embedding process is illustrated in Fig.1.

The Owner's photo is first scanned into the computer. The scanned photo will be the host image for the embedding algorithm. The watermark is a sequence of personal information (a sequence consisting of combination of characters and numbers is used here). The embedding algorithm is based on the polarity of Hadamard transform coefficients, that determines the insertion of the watermark sequence into the host image. The watermarked image and a secret key, are then generated. The watermarked photo is printed on the owner's security document or card. The retrieval and verification process, as depicted in Fig. 2, is performed on the scanned photo of an inspected card for authentication.

\section{B. Print-and-scan process}

There is currently very limited research has being performed on the print-and-scan process. After a digital image is printedand-scanned, various distortions are introduced. Distortion could occur in both the pixel values and the geometric boundary of the rescanned image [8]. 


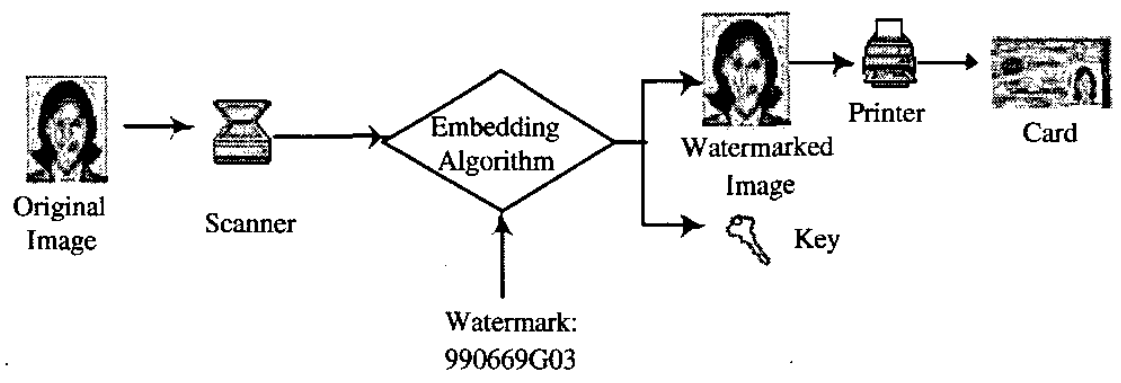

Fig. 1. Embedding process.

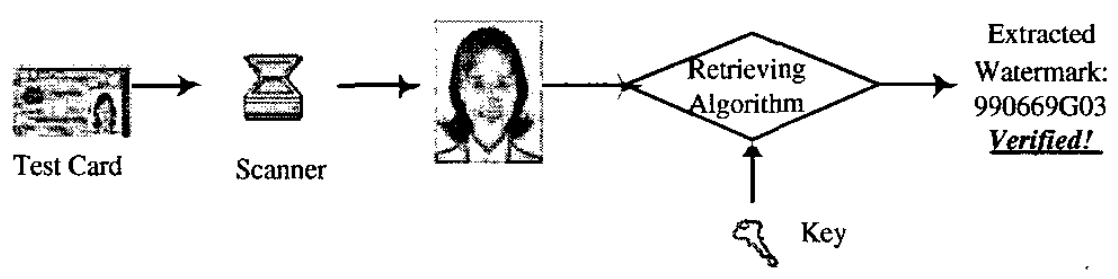

Fig. 2. Retrieval and verification process.

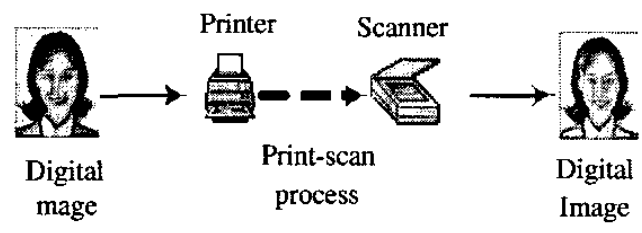

Fig. 3. Print-and-scan process.

Typical distortions of pixel values could be caused by the luminance, contrast, gamma correction, chrominance variations and the blurring of adjacent pixels. Distortion of the geometric boundary in a DAD (digital-analogue-digital) process is caused by rotation, scaling and cropping. The print-and-scan process is shown in Fig.3.

The PS process could be modeled and explained by dividing into two scenarios. For the first scenario, rotation and cropping are included in the PS process. Scenario 1 PS is usually performed by an adversary, thus such PS is considered as a malicious combined attack. While in Scenario 2, rotation and cropping are not taken into account. To overcome Scenario 1 PS, techniques capable of conquering rotation, scaling and translation (RST) must be simultaneously included. The RST attacks, however, still remain an unsolved problem in watermarking, although some techniques were proposed in the literature [4]. In our system, the PS is considered as an inherent system distortion rather than a malicious attack, thus Scenario 2 PS process is involved.
C. Spread spectrum based watermark embedding and retrieval

In this section, the proposed watermark embedding and retrieval algorithm is presented.

1) Watermark embedding algorithm: The spread spectrum based watermark embedding algorithm is depicted in Fig.4.

First, the watermark bit, either 1 or 0 , is expanded into a binary sequence in the following manner: if the watermark bit is 1 , then it is represented by data $\mathbf{w}_{m 1}$ : $\{1,-1,1,-1,1,-1,1,-1, \ldots\}$ and the length of this sequence is $m$; if the watermark bit is 0 , then it is represented by data $\mathbf{w}_{m 0}:\{-1,1,-1,1,-1,1,-1, \ldots\}$ and the length of this sequence is also $m$.

Then we transform the original image. A Hadamard transform is used in our algorithm, due to its simplicity and ease for hardware implementation [5]. For one bit of watermark, that has been expanded into a sequence with length of $m$, we need to use the same amount of coefficients for insertion. The embedding process can be divided into two passes:

(1)Polarity modulation;

(2)Amplitude modulation. 


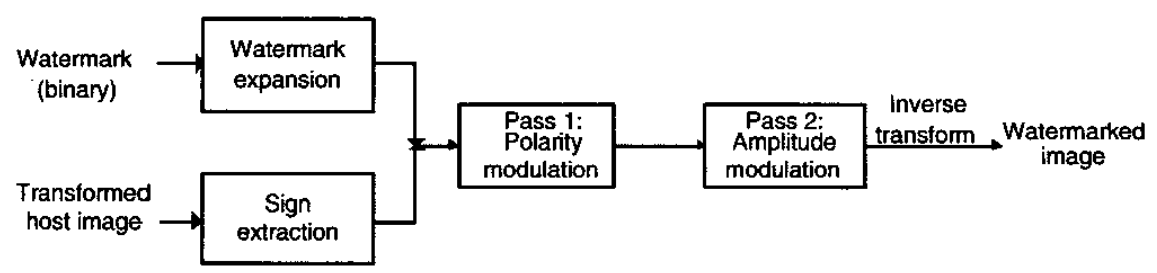

Fig. 4. Block diagram of watermark embedding algorithm.

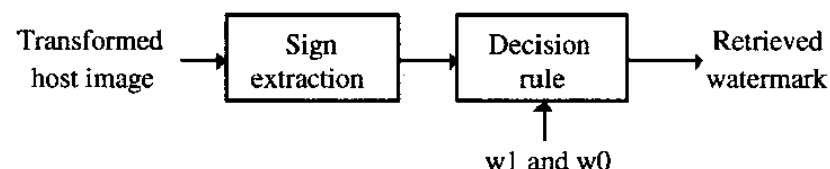

Fig. 5. Block diagram of watermark extraction algorithm.

Consider that we embed one watermark bit. At first, we obtain the polarity sequence of the selected transform coefficients, as follows:

$$
s_{m}[i]=\left\{\begin{array}{lll}
1, & \text { if } & c[i] \geq 0 \\
0, & \text { if } & c[i]<0
\end{array}\right.
$$

where $c[\mathrm{i}](1 \leq i \leq m)$ denotes selected coefficients.

For the first pass, we modulate the coefficients' polarities in order to make the correlation value between $s_{m}$ and $\mathbf{w}_{m 1}$ or $\mathbf{w}_{m 0}$ satisfying the following equation:

$$
\operatorname{corr}= \begin{cases}\frac{\mathbf{s}_{m} \mathbf{w}_{m 1}^{\prime}}{\sqrt{\left(\mathbf{S}_{m} \mathbf{S}_{m}^{\prime}\right)\left(\mathbf{w}_{m 1} \mathbf{w}_{m 1}^{\prime}\right)}}>\mathrm{T}_{1}, & \text { if } \quad \text { wmk bit }=1 \\ \frac{\mathbf{S}_{m} \mathbf{w}_{m 0}^{\prime}}{\sqrt{\left(\mathbf{S}_{m} \mathbf{S}_{m}^{\prime}\right)\left(\mathbf{w}_{m 0} \mathbf{w}_{m 0}^{\prime}\right)}}>\mathrm{T}_{1}, & \text { if } \quad \text { wmk bit }=0\end{cases}
$$

where $T_{1}$ denotes certain threshold for the correlation. In our experiments, we set $T_{1}$ as 0.3 . If the original polarity sequence does not satisfy Equation (2), some of the coefficients' polarities would be changed until the modified polarity sequence satisfies the condition. However, if we change the polarities of the coefficients having large amplitude, the image quality is easily degraded. Thus, the polarities of those transform coefficients having smaller amplitude should be changed with higher priority.

In the amplitude modulation pass, we increase the amplitude of all the selected coefficients up to the prescribed threshold $T_{2}$, with the polarity of coefficients maintained. After a watermarked image has gone through some distortion channels, the transform coefficients would be altered. Such alteration, however, is generally within a certain range [9]. Therefore, if the amplitude of a coefficient is increased to be greater than the range, it has a higher probability to maintain its polarity under certain attacks such as JPEG compression, median filtering, although its amplitude would still be altered. The equation for amplitude modulation is defined as follows.

$$
c^{\prime}=\operatorname{sign}(c)\left[|c|+\left(n+1-\left\lceil\frac{|c| n}{\mathrm{~T}_{2}}\right\rceil\right) \frac{\mathbf{T}_{2}}{n}\right]
$$

where $n$ is defined as a certain step size, \lceil\rceil is the ceil function, and $c$ and $c^{\prime}$ denote original coefficients and modulated coefficents, respectively. We define the $\operatorname{sign}(c)$ as follows:

$$
\operatorname{sign}(c)= \begin{cases}1, & \text { if } c \geq 0 \\ -1, & \text { if otherwise }\end{cases}
$$

By using Equation (4), the coefficients' amplitudes are increased to the range $\left[T_{2}, T_{2}+\frac{T_{2}}{n}\right]$.

2) Watermark retrieval algorithm: To extract the watermark, we again perform a Hadamard transform to the scanned photograph, and the pseudo-random generator seed is obtained from the secret key to find the locations of the coefficients containing the watermark. We define these coefficients as $\mathbf{c}_{m}^{*}$ . We extract the polarities of $\mathbf{c}_{m}^{*}$ and denote polarity sequence as $\mathbf{s}_{m}^{*}$. The extraction is performed by the correlation between $\mathbf{s}_{m}^{*}$ and $\mathbf{w}_{m 1}$ or $\mathbf{w}_{m 0}$,

$$
\begin{aligned}
& \operatorname{corr}_{1}^{*}=\frac{\mathbf{s}_{m} \mathbf{w}_{m 1}^{\prime}}{\sqrt{\left(\mathbf{s}_{m}^{*} \mathbf{s}_{m}^{*^{\prime}}\right)\left(\mathbf{w}_{m 1} \mathbf{w}_{m 1}^{\prime}\right)}} \\
& \operatorname{corr}_{0}^{*}=\frac{\mathbf{s}_{m} \mathbf{w}_{m 0}^{\prime}}{\sqrt{\left(\mathbf{s}_{m}^{*} \mathbf{s}_{m}^{*^{\prime}}\right)\left(\mathbf{w}_{m 0} \mathbf{w}_{m 0}^{\prime}\right)}}
\end{aligned}
$$

$c o r r_{1}^{*}$ and $c o r r_{0}^{*}$ measure the possibility that the embedded bit is 1 and 0 , respectively. The decision rule of the watermark bit is given as follows. $k$ denotes the extracted watermark bit.

$$
k= \begin{cases}1, & \text { if } \quad \operatorname{corr} r_{1}^{*} \geq \operatorname{cor} r_{0}^{*} \\ 0, & \text { if } \quad \operatorname{cor} r_{0}^{*}>\operatorname{cor} r_{1}^{*}\end{cases}
$$

For detection, the detector does not require both the original image and the watermark. Such watermarking schemes are called public watermarking schemes or blind watermarking schemes [10]. 


\begin{tabular}{|c|c|c|c|c|}
\hline $\begin{array}{c}\text { Original } \\
\text { photo } \\
(180 \times 130)\end{array}$ & $\begin{array}{c}\text { Watermarked } \\
\text { photo } \\
(180 \times 130)\end{array}$ & $\begin{array}{c}\text { Printed- } \\
\text { scanned } \\
\text { photo } \\
(215 \times 160)\end{array}$ & Watermark & $\begin{array}{c}\text { Verification } \\
\text { (Yes/No) }\end{array}$ \\
\hline & & & AAAAA1111 & Yes \\
\hline & & & BBBBB2222 & Yes \\
\hline & & & ССССС 3333 & Yes \\
\hline & & $x^{2}+2-5=0$ & & \\
\hline
\end{tabular}

Fig. 6. Samples of experimental results.(The characters used here are not real identity numbers.)

\section{EXPERIMENTAL RESULTS}

In our experiments, we used a common laser printer (HP LaserJet 4MV) and scanner (CanoScan N1220U), with resolutions set to $170 \mathrm{dpi}$ (dots per inch) and $200 \mathrm{dpi}$, respectively. For subblocking the image, an $8 \times 8$ Hadamard transform was used. The watermark expansion length was set to 8 , and $T_{1}$ in polarity modulation and $T_{2}$ in amplitude modulation were set to 0.3 and 1.5 , respectively. A tradeoff between the robustness and image quality was determined in selecting $T_{1}$ and $T_{2}$. These empirical values were obtained based on both the acceptable image quality and robustness against the PS process. We tested our authentication system for 105 different face photographs and the success rate was better than $96 \%$. The failures were mainly due to manual misalignment, because our algorithm was not so resistant to rotation and translation attacks. Some examples of the experimental results are listed in Fig. 6.

\section{CONCLUSION}

We proposed a card authentication system based on digital watermarking. A linkage between the bearer's photograph and his/her personal information is established through superimposing the personal information directly into the photograph as a watermark. As such, photograph substitution frauds could be prevented using this approach. The watermarking algorithm was based on the polarity of Hadamard transform coefficients, that could invisibly insert a 9-character watermark into smooth photographs of size $180 \times 130$ pixels. This algorithm successfully survived with a success rate achieved better than $96 \%$ for
105 different face photographs. the print-and-scan process. The proposed system can be used to authenticate student cards, passports, driving licenses and other security documents or cards.

\section{REFERENCES}

[1] R. B. Wolfgang and E. J. Delp, "Fragile watermarking using the VW2d watermark", Proceeding of the SPIEIS\&T Intemational Conference on Security and Watermarking of Multimedia Contents, vol. 3657, pp. 204213, Jan. 1999.

[2] J. Hu, J. Huang, D. Huang and Y. Q. Shi, "Image fragile watermarking based on fusion of multi-resolution tamper detection," IEE Electronic Letters,vol. 38, no. 24, pp 1512-1513, Nov. 2002.

[3] D. Kundur and D. Hatzinakos, "Digital watermarking for telltale tamperproofing and authentication," Proceedings of the IEEE Special Issue on Identification and Protection of Multimedia Information, vol. 87, no. 7, pp. 1167-1180, July 1999.

[4] C.-Y. Lin, M. Wu, J. A. Bloom, I. J. Cox, M. L. Miller, and Y. M. Lui, "Rotation, scale, and translation resilient watermarking for images," IEEE Trans. Image Processing, vol. 10, no. 5, May 2001, pp. $767-782$.

[5] A. T. S. Ho, J. Shen, and S. H. Tan. "Robust digital image-in-image watermarking algorithm using the fast Hadamard transform," SPIE's 47th Annual Meeting on Optical Science and Technology, July 2002, Seattle, USA.

[6] A. T. S. Ho, J. Shen, H. P. Tan, and J. Woon, "Security-printing authentication using digital watermarking," Electronic Imaging, vol. 13, no.1, Jan. 2003.

[7] J. Mercer, Authentication News, 5 (9/10), 2001.

[8] C.-Y. Lin and S.-F. Chang, "Distortion modeling and invariant extraction for digital image print-and-scan process," Intl. Symp. on Multimedia Information Processing, Taipei, Taiwan, Dec. 1999.

[9] F. Shu, A. T. S. Ho and J. Shen, "Digital Watermarking Techniques Using Polarity of Transform Coefficients," Accepted by IASTED International Conference on Signal and Image Processing,Aug. 2003, Honolulu, USA.

[10] S. Katzenbeisser and A. P. F. Petitcolas, Information Hiding Techniques for Steganography and Digital Watermarking, Artech House, 2000. 